\title{
Nanoplate field-effect capacitive (bio-)chemical sensor array based on SOI structure
}

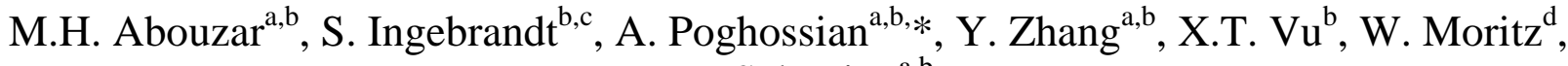 \\ M.J. Schöning ${ }^{\mathrm{a}, \mathrm{b}}$ \\ ${ }^{a}$ Institute of Nano- and Biotechnologies (INB), Aachen University of Applied Sciences, Campus \\ Jülich, 52428 Jülich, Germany \\ ${ }^{b}$ Institute of Bio- and Nanosystems (IBN-2), Research Centre Jülich GmbH, 52425 Jülich, Germany \\ ${ }^{c}$ Department of Informatics and Microsystem Technology, University of Applied Sciences Kaiserslautern, Campus Zweibrücken, \\ 66482 Zweibrücken, Germany \\ ${ }^{d}$ Institute of Chemistry, Humboldt University Berlin, 12489 Berlin, Germany
}

\begin{abstract}
An array of individually addressable nanoplate field-effect capacitive (bio-)chemical sensors based on an SOI (silicon-oninsulator) structure has been developed for multi-parameter detection. An isolation of the individual capacitors was achieved by forming a trench in the top Si layer with various thicknesses of 30-350 $\mathrm{nm}$. The feasibility of the proposed approach has been demonstrated by realising sensors for the detection of $\mathrm{pH}$ as well as for the label-free electrical monitoring of adsorption and binding of charged macromolecules.
\end{abstract}

Keywords: field-effect; pH sensor; SOI; label-free detection; charged macromolecules; polyelectrolyte

\section{Introduction}

Field-effect capacitive EIS (electrolyte-insulator-semiconductor) sensors have been recognised as very promising tool for the detection of various (bio-)chemical parameters (see e.g., recent reviews ${ }^{1,2}$ ). Although capacitive EIS single sensors are simple in layout and easy in fabrication, nevertheless, the integration of multiple EIS sensors onto one silicon chip is problematic due to difficulties in preparation of separate, electrically isolated individual EIS capacitors. In early experiments, it has been demonstrated that the electrical isolation of one-chip integrated multiple ISFET-based (ion-sensitive field-effect transistor) (bio-)chemical sensors can be achieved using silicon-on-insulator (SOI) wafers $^{3-5}$. More recently, the SOI technology has been applied for the fabrication of a (bio-)chemically sensitive nanowire-transistor array $^{6}$ and silicon thin-film resistors ${ }^{7}$. In these structures, the bottom Si serves as substrate while the top thin Si layer is used to fabricate the transducer structure.

In this work, an array of individually addressable nanoplate field-effect capacitive EIS sensors based on an SOI

\footnotetext{
* Corresponding author. Tel.: +49-2461-612605; fax: +49-2461-612940.

E-mail address: a.poghossian@fz-juelich.de
} 
structure (EISOI) with different thicknesses of the top $\mathrm{Si}$ layer has been developed for multi-parameter (bio-)chemical sensing for the first time. The realised nanoplate capacitive sensors have been exemplarily tested for the $\mathrm{pH}$ measurement as well as for the label-free detection of charged macromolecules, like polyelectrolytes (PE).

\section{Experimental}

\subsection{EISOI sensor fabrication}

Four groups of a field-effect capacitive sensor array with various thicknesses (350, 170, 60 and $30 \mathrm{~nm})$ of the top Si layer were fabricated from an SOI wafer (Soitech, France) with a $360 \mathrm{~nm}$ thick top p-Si layer on a $400 \mathrm{~nm}$ thick buried $\mathrm{SiO}_{2}$ layer (Fig. 1). Each array consists of four nanoplate capacitive structures. For the preparation of the field-effect capacitors with various thicknesses of the top Si layer (Fig. 1b), the top Si layer has been partially oxidised by means of thermal wet oxidation. An isolation of the individual capacitors was achieved by forming a trench in the top Si layer. As an example, Fig. 2 shows the scanning electron microscopy picture of a layer structure with a $350 \mathrm{~nm}$ thick top $\mathrm{Si}$ layer and a $30 \mathrm{~nm} \mathrm{SiO}$ layer as gate insulator. Thus, this fabrication technology allows addressable as well as simultaneous biasing of multiple field-effect capacitors on the same SOI chip.

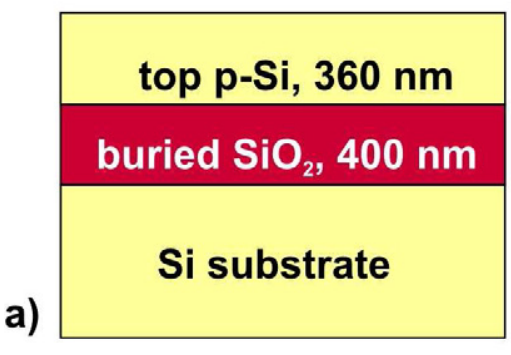

b)

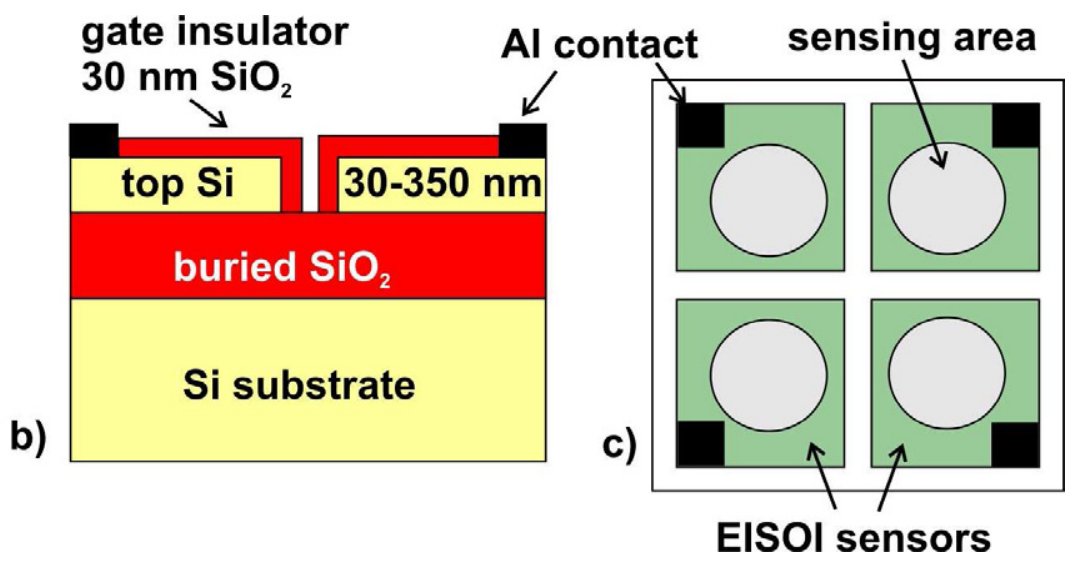

Fig. 1. Schematic cross section of the SOI wafer (a), capacitive EISOI sensor (b) and top view of the sensor array (c).

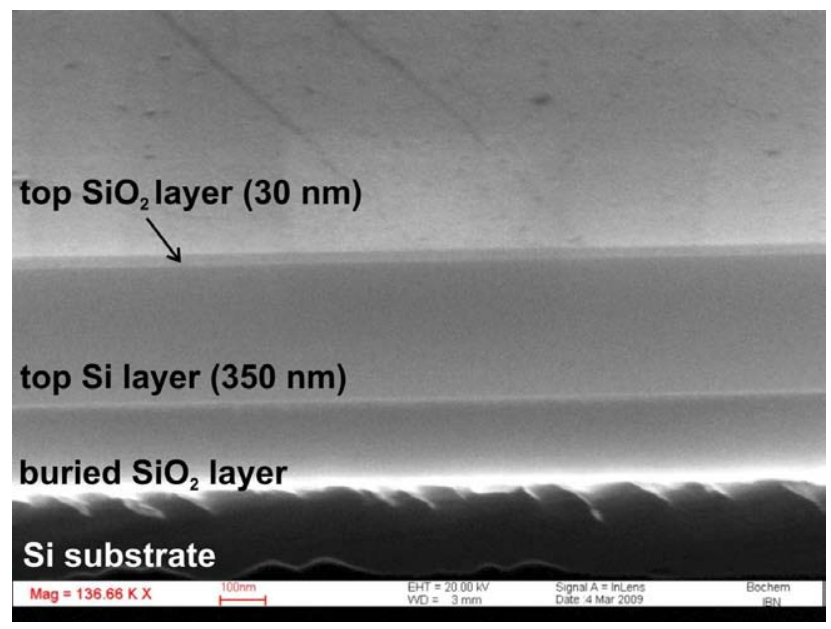

Fig. 2. Scanning electron microscopy picture of the EISOI sensor layer structure. 


\subsection{EISOI measurement set-up}

The developed EISOI sensors have been characterised by means of constant-capacitance (ConCap) method using an impedance analyser (Zahner Elektrik) (see measurement set-up in Fig. 3). For operation, a DC polarisation voltage is applied via a conventional $\mathrm{Ag} / \mathrm{AgCl}$ electrode reference electrode to set the working point of the EISOI sensor, and a small $\mathrm{AC}$ voltage $(20 \mathrm{mV}, 30 \mathrm{~Hz})$ is applied to the system in order to measure the capacitance of the sensor.

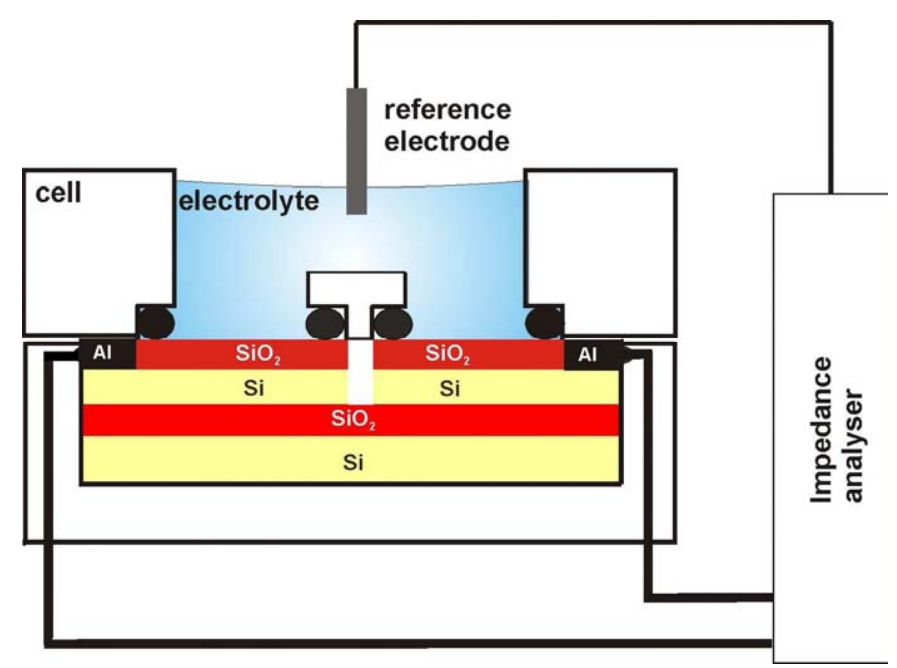

Fig. 3. Measuring set-up for the electrochemical characterisation of the EISOI sensor array (schematically).

For the measurements, the EISOI sensor was mounted into a home-made measuring cell, sealed by an O-ring and contacted on its front side by the electrolyte and a reference electrode, and on its top-silicon side by a gold-plated pin. The contact area of the EISOI sensor with the solution was about $0.126 \mathrm{~cm}^{2}$.

\section{Results and discussion}

\section{1. $p H$ sensitivity of the EISOI sensor}

For the $\mathrm{pH}$ measuring procedure, about $1 \mathrm{ml}$ of the working buffer (Titrisol buffer) was applied to the EISOI gate surface, and the sensor output signal was recorded. The EISOI sensors show an average $\mathrm{pH}$ sensitivity of 36 $\mathrm{mV} / \mathrm{pH}$ (evaluated from the ConCap response) in the range of $\mathrm{pH} \mathrm{3-11} \mathrm{that} \mathrm{is} \mathrm{in} \mathrm{agree} \mathrm{with} \mathrm{reported} \mathrm{results}$ $(34-40 \mathrm{mV} / \mathrm{pH})$ for capacitive EIS structures and ISFETs with a $\mathrm{SiO}_{2}$ layer as a gate insulator ${ }^{8}$.

\subsection{EISOI sensor for the detection of charged macromolecules}

The PE multilayer (maximum number of PE layers was 14) was obtained using the layer-by-layer technique by consecutive adsorption of positively charged PAH (Poly (allylamine hydrochloride)) and negatively charged PSS (Poly (sodium 4-styrene sulfonate) ) from the respective PE solution $(50 \mu \mathrm{M}$ PSS or PAH, unbuffered $0.01 \mathrm{M} \mathrm{NaCl}$, $\mathrm{pH}$ 5.3). We started the formation of the PE multilayer onto the sensor surface with positively charged PAH. Alternating potential shifts $(8-55 \mathrm{mV})$ have been observed after the adsorption of each PSS and PAH layer onto the $\mathrm{SiO}_{2}$ surface (see Fig. 4). The direction of the shift depends on the sign of the charge of the terminating PE layer. Moreover, the potential shifts have a tendency to decrease with increasing the number of PE layers ${ }^{9-11}$. 


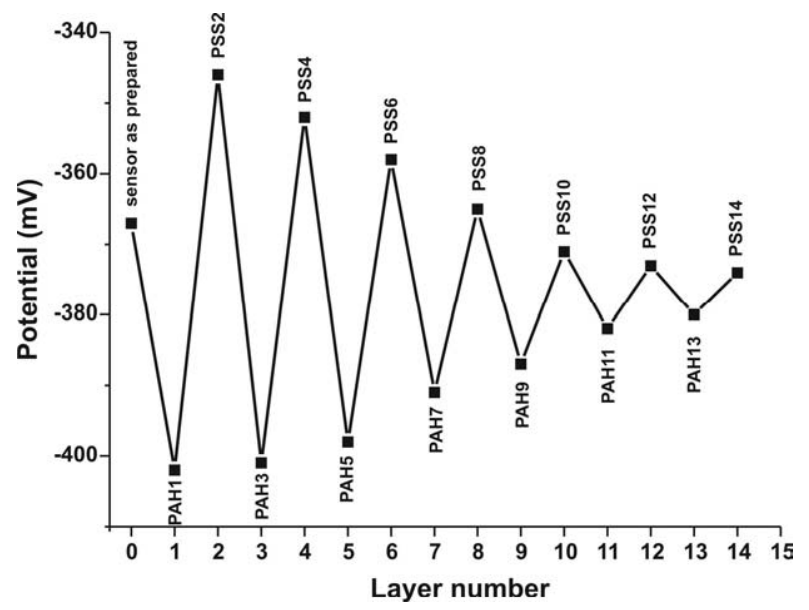

Fig. 4. Detection of layer-by-layer adsorption of polyelectrolytes with an EISOI sensor; alternating potential changes with tendency to decrease with increasing the layer number have been observed after the adsorption of each polyelectrolyte layer.

\section{Acknowledgements}

The authors gratefully thank H.P. Bochem for technical support.

\section{References}

1. Poghossian A, Ingebrandt S, Offenhäusser A, Schöning MJ. Feld-effect devices for detecting cellular signals. Semin Cell Dev Biol 2009;20:41-8.

2. Schöning MJ, Poghossian A. Detection of charged macromolecules by means of field-effect devices (FEDs): possibilities and limitations. In: Zhang X, Ju H, Wang J, editors. Electrochemical Sensors, Biosensors and their Biomedical Applications, Amsterdam (The Netherlands), Elsevier; 2008, p. 187-212.

3. Poghossian A. Method of fabrication of ISFETs and CHEMFETs on an $\mathrm{Si}_{-} \mathrm{SiO}_{2}$-Si structure. Sens. Actuators B 1993;14:653-4.

4. Poghossian A. Method of fabrication of ISFET-based biosensors on an $\mathrm{Si}_{-} \mathrm{SiO}_{2}$-Si structure. Sens. Actuators B 1997;44:361-4.

5. Moreno L, Merlos A, Abramova N, Jimenez C, Bratov A. Multi-sensor array used as an "electronic tongue" for mineral water analysis. Sens. Actuators B 2006;116:130-4.

6. Vu XT, Eschermann JF, Stockmann R, Ghosh Moulick R, Offenhäusser A, Ingebrand S. Top-down processed silicon nanowire transistor arrays for biosensing. Phys. Stat. Sol. (a) 2009;206:426-434.

7. Neff PA, Serr A, Wunderlich BK, Bausch AR. Label-free electrical detection of trypsin activity by a silicon-on-insulator based thin film resistor. ChemPhysChem 2007;14:2133-7.

8. Poghossian A, Schöning MJ. Silicon-based chemical and biological field-effect sensors. In: Grimes CA, Dickey EC, Pishko MV, editors. Encyclopedia of Sensors, Stevenson Ranch (USA): American Scientific Publishers; 2006, p. 463-533.

9. Poghossian A, Abouzar MH, Sakkari M, Kassab T, Han Y, Ingebrandt S, et al. Field-effect sensors for monitoring the layer-by-layer adsorption of charged macromolecules. Sens. Actuators B 2006;118:163-170.

10. Poghossian A, Ingebrandt S, Abouzar MH, Schöning MJ. Label-free detection of charged macromolecules by using a field-effect-based sensor platform: Experiments and possible mechanisms of signal generation. Appl. Phys. A. 2007;87:517-524.

11. Poghossian A, Abouzar MH, Amberger F, Mayer D, Han Y, Ingebrandt S, et al. Field-effect sensors with charged macromolecules: Characterisation by capacitance-voltage, constant-capacitance, impedance spectroscopy and atomic-force microscopy methods. Biosens. Bioelectron. 2007;22:2100-7. 